\title{
Extinctions by catastrophe?
}

\section{The suggestion that the disappearence of species from the surface of the Earth has been periodic has stimulated contradictory explanations and also a diversity of ways of dealing with information.}

DOES the periodic extinction of species on the surface of the Earth arise because the Sun has a previously unknown dwarf companion which disturbs the Solar System's cometary cloud, or because the Solar System as a whole oscillates above and below the plane of the Galaxy? And, in any case, how solid is the assertion that during the past 250 million years, there has been a statistical regularity in the timing of the disappearance of large numbers of species from the surface of the Earth?

These questions are certain to be provoked by the group of five articles appearing on pages 709 to 720 of this issue. Professor Anthony Hallam on page 686 gives some of the reasons why openmindedness on the last question is still appropriate. Readers who are perplexed by the stark contradiction between the two explanations now advanced should not, however, blame the authors of the two hypotheses but, rather, the conventions, acceptable and otherwise, which regulate, and sometimes fail to regulate, such developments.

One obvious difficulty is that all five of the articles now published refer in one way or another to an article by David M. Raup and J. John Sepkoski, of the University of Chicago, which appeared only in the February issue of the Proceedings of the National Academy of Sciences (81, $801-805 ; 1984)$. That publication is itself the culmination of a long process - a detailed compilation by Sepkoski (Milwaukee Contr. Biol. Geol. 51, 125; 1982) of what is known about the dates of the extinction of species on the surface of the Earth, partial consideration by one or other of the authors of the significance of this mass of data and eventually their conviction that there is probably a periodicity of 26 million years in the occurrence of extinctions. This conclusion seems to have been formed in their minds by May last year, when one of the authors described the result at one of the Dahlem conferences in Berlin. The article in which the conclusion is embodied was submitted for publication the following October, and has now appeared in print.

There is nothing remarkable about this sequence of events. Raup and Sepkoski are not the first to have suggested that there may be a periodicity in the pattern of species extinction, and have been properly cautious in putting forward their new interpretation of the data. The problem is notoriously difficult. The fossil record is so poor that it is not possible to tell when individual species became extinct. It is necessary instead to work with taxonomic families, but even then it is possible to place their extinction only somewhere within the confines of a stratigraphical stage, of which 39 , with different durations, are placed by international convention within the past 250 million years.

One obvious statistical pitfall is that the conventionally accepted pattern of stratigraphical stages might itself conceal a periodicity. Raup and Sepkoski break new ground in the analysis of data-sets thought to harbour periodicity by their use of computer-based tests of the reality of their inferred period of 26 million years. The authors themselves acknowledge that the conclusion that species extinction is a periodic phenomenon will be jeopardized if the stratigraphical time scale proves to be incorrect (Hallam, op. cit.).

So why have Raup and Sepkoski prevailed when others advocating periodic extinctions have done less well? Obviously it has helped that their statistical analysis has been done in a way that shows them to be fully aware of the pitfalls. But it is proper to acknowledge that the intellectual climate has changed in favour of catastrophism, especially now that Luiz and Walter Alvarez appear to have proved their original case that the massive extinction at the end of the Cretaceous period was caused by the impact of some extraterrestrial object (see Science 223, 1174-1186; 1983). Indeed, Raup and Sepkoski themselves say at the end of their article that a biological explanation of their periodicity "seems incredible" and that they favour "extraterrestrial causes".

Extraterrestrial explanations they have now stimulated in abundance, partly because preprints of their article appear to have been freely circulated in advance of publication. What seems now to have happened is that the statement by two careful investigators that extraterrestrial phenomena may be responsible for mass species extinctions has been taken by the astrophysicists as a signal to put forward models to account for what may have occurred.

Two important issues arise from this, the first of them concerned with the process of professional communication and, in particular, with the great confusion that may be caused by the widespread circulation of preprints. This practice, usually intended as a courtesy to colleagues elsewhere (and welcomed by the recipients except when the effect is to pre-empt a patch of intellectual territory), can also be thoroughly unhelpful to other people. The most obvious complaint against the system is that it is discriminatory, excluding from the circle of those in the know people who happen not to be on the authors' mailing list. On this occasion, the first accounts of models to explain periodic extinction were so swiftly on their way to Nature that, if revision of their content had not been recommended, it would have been possible for them to have appeared in print before Raup and Sepkoski.

Confusion has been further augmented by the circumstance that a full account of the article by Davis et al. (see p.715) appeared in the San Francisco Chronicle în mid-February, and that a workshop on the subject (organized by Dr Luiz Alvarez) was held at Berkeley, California on 3 and 4 March, and has since been agnostically reported (Science 223, 2178; 1984). (A representative of Nature was invited but could not attend, perhaps, in the circumstances, fortunately.) One result of these developments is that criticisms of some aspects of the group of papers now published are already flowing in.

Developments such as these constitute a kind of nonsense. In the normal course of events, it is entirely proper and indeed essential that people should talk about their discoveries at conferences, and give their colleagues advance notice of what they plan to publish, while the occasional publication of the details of some new discovery in the general press does not spell the end of the world. But the concatenation of all these events can create serious doubts in people's minds about the proper attribution of novel ideas to the participants.

The second issue raised by the two groups of articles now published is more interesting because it draws attention to the difference of what may be called style that appears to distinguish astrophysicists from, say, biologists. The models now proposed for periodic extinctions are lent credibility only because Raup and Sepkoski have put forward a conclusion that cries out for an explanation. Reflection will show that astrophysicists often have no other way in which to make progress. The fact that, on this occasion, two contrasting models seem to be equally plausible must, for the time being, be counted an unlucky accident.

John Maddox 